\title{
EL TURISMO DE SALUD EN EL ESTADO DE HIDALGO, MÉXICO. PROPUESTAS PARA CONSOLIDARLO
}

\author{
HEALTH TOURISM IN THE STATE OF HIDALGO, MEXICO. \\ PROPOSALS FOR ITS CONSOLIDATION
}

\author{
Maximiliano Gracia Hernández \\ Colegio del Estado de Hidalgo y Universidad La Salle, México
}

\section{RESUMEN}

El objetivo de este trabajo fue determinar las fortalezas, oportunidades, debilidades y amenazas que tiene el corredor turístico de balnearios de Ixmiquilpan - Tasquillo, lo cual se logró detectar a partir de un estudio de campo in situ, acompañado de una serie de entrevistas aplicadas a visitantes y turistas a su salida del balneario. La técnica de la investigación se basó en la aplicación de cuarenta entrevistas. Derivado del trabajo de campo se muestra los resultados y por ende la realidad actual de los balnearios de aguas termales del corredor Ixmiquilpan - Tasquillo en el estado de Hidalgo. Los resultados obtenidos muestran que los balnearios del mencionado corredor no cuentan con los servicios secundarios que les permita ser explotados con eficacia y eficiencia. Se debe pensar en el cambio y en la innovación permanente y, es que las nuevas necesidades y deseos de la demanda de hoy son diferentes a lo que se demandaba ayer.

Palabras clave: Termal, turismo de salud, Hidalgo, balnearios, aguas termales.

\begin{abstract}
The target of this study was to determine the strengths, weaknesses, opportunities and threats that there has the tourist area of resorts Ixmiquilpan - Tasquillo, the previous thing managed to be discovered from a field study in situ, accompanied by a series of interviews answered by visitors and tourists to its exit of the tourist area. The research technique was based on the application of forty interviews. Derivative of the investigation we show the results and the reality of the spas in the tourist area of Ixmiquilpan-Tasquillo in Hidalgo State. The results show that the tourist area Ixmiquilpan - Tasquillo not has the secondary services that allow them to be exploited effectively and efficiently. It is necessary to think about the change and about the permanent innovation and, the fact is that the new needs and desires of the today demand it is different from what was demanded yesterday.
\end{abstract}

Keywords: Thermal, tourism of health, Hidalgo, spas, thermal waters 


\section{Introducción}

Por nivel de ingresos el turismo representa para México el tercer lugar nacional, sin embargo, ese lugar privilegiado del turismo dentro de la economía no lo es todo, porque este sector tiene un efecto multiplicador positivo en el ingreso de los que participan dentro de él, y es que los visitantes y turistas pagan alimentos, abordan taxis, disfrutan de tours, acuden a bares y discotecas, compran regalos y artesanías, gastan para el hospedaje, dan propinas, etc., de ahí su importancia no solo como efecto económico, sino también por las externalidades positivas que ese sector genera a quienes participan dentro de él en forma directa e indirecta.

Existen Estados de la república mexicana que basan parte de su desarrollo económico en el sector turístico, por ejemplo, los estados de Quintana Roo, Yucatán, Chiapas y Oaxaca; no obstante, también hay regiones en el país con una gran cantidad de zonas turísticas poco explotadas o en proceso de explotación, es el caso del estado de Hidalgo, región con una gran variedad de zonas culturales, recreativas, rurales, históricas y naturales que son poco explotadas.

En el marco antes señalado, no podemos obviar el tema del turismo de salud en el estado de Hidalgo, el cual tiene gran importancia dentro del contexto nacional, particularmente por la existencia de balnearios de aguas termales en algunas regiones del Estado (la palabra terma se aplica al agua que brota de la tierra y cuya temperatura es superior a la del medio ambiente).

En el estado de Hidalgo la zona de balnearios con aguas termales debe presentar una alternativa a otro tipo de turismo convencional y, es que además de la diversión que representa para toda la familia, puede llegar a ser un centro de salud para todos. Las aguas termales y, particularmente la infraestructura de balnearios son el principal atractivo turístico de la región Ixmiquilpan - Tasquillo. El descanso y la diversión son dos de las principales razones para que el turista visite ese destino.

El objetivo de este trabajo es determinar las fortalezas, oportunidades, debilidades y amenazas que tiene el corredor turístico de balnearios de Ixmiquilpan - Tasquillo, por medio de un estudio de campo in situ, acompañado de una serie de entrevistas aplicadas a visitantes y turistas.

La investigación considera tres tipos de estudios a realizar: exploratorio, descriptivo y explicativo de propuesta. La parte exploratoria permitirá desarrollar una investigación que nos ofrezca una visión general de tipo aproximativo respecto a la realidad de los balnearios de Ixmiquilpan - Tasquillo; la parte descriptiva busca especificar las características de los balnearios y los servicios prestados; y, la parte explicativa de la propuesta mide diversos aspectos, dimensiones o componentes del fenómeno a investigar, es decir, aborda las ventajas competitivas y comparativas del objeto de estudio, así como también muestra las fortalezas, oportunidades, debilidades y amenazas de las condiciones actuales.

La técnica de la investigación se basó en la aplicación de cuarenta entrevistas con el respectivo análisis de sus resultados. Derivado del trabajo de campo se muestra la realidad actual de los balnearios de aguas termales del corredor Ixmiquilpan - Tasquillo. 
El trabajo se estructura de la siguiente manera: En el primer apartado se aborda el origen de los balnearios de aguas termales y se reflexiona acerca del termalismo, la salud y el turismo; en el segundo apartado, se determinan, con base en el trabajo de campo, las fortalezas, oportunidades, debilidades y amenazas del turismo de salud dentro del corredor turístico Ixmiquilpan - Tasquillo en el estado de Hidalgo. Por último, se proponen los retos y acciones para fortalecer el turismo de salud en la región de estudio.

\section{Las aguas termales y sus beneficios a lo largo de la historia}

A lo largo de la historia las civilizaciones han utilizado las aguas termales como una medida terapéutica, existen construcciones en las cuales se realizaban baños con aguas termales que datan de hace cientos de años.

Según los anales de la historia, el turismo de salud representa uno de los más antiguos que se conocen. Desde el siglo V a. C. fueron los griegos y posteriormente los romanos quienes otorgaron al balneario un enfoque cultural (Fernández, 1991). La tradición de los balnearios continuó por los árabes, esta cultura aprovechó las termas romanas. Durante las Cruzadas los baños de aguas termales florecieron con mayor fuerza y, es que se pensó que estas eran una solución para recuperar a los heridos por las guerras.

En años recientes el surgimiento de grandes emporios turísticos focalizados en la integración humanonatural, el regreso a medios tradicionales de salud y a la creciente oferta internacional de servicio médicos internacionales, hicieron que en las últimas décadas se diera el boom en el resurgimiento del turismo de salud. (Balderas, 2014)

\section{Termalismo, salud y turismo}

En el momento actual el termalismo se considera para:

- Tratamientos de enfermedades.

- Prevención y el mantenimiento del funcionamiento adecuado del organismo (San José, 2000).

Progresivamente, en los últimos años, se observa interés por el termalismo bajo un escenario turístico.

La Organización Mundial de la Salud declaró en 1986 a las aguas termales como una herramienta para lograr buena salud. Hoy existe una disciplina llamada hidrología médica, está comprobado que el agua termal incrementa la temperatura del cuerpo y con ello elimina gérmenes, incrementa la presión hidrostática del cuerpo y se logra aumentar la circulación sanguínea y la oxigenación. Este aumento en la temperatura ayuda a disolver y eliminar las toxinas del cuerpo, se estimulan las defensas; se relajan los músculos; se combate el estrés y las enfermedades de la piel y respiratorias leves.

El aprovechamiento de las aguas termales no requiere el empleo de energía para elevar la temperatura del agua, no excluye que el agua tenga otros usos y brinde otros servicios. Desde los puntos de vista económico y ecológico, el aprovechamiento de las aguas termales genera un servicio recreativo provisto por el funcionamiento 
del ecosistema, el cual se conoce como servicio ambiental recreativo, definido como los beneficios que la gente obtiene de los ecosistemas (Wakter et al., 2004, citado en Ortíz et al., 2014).

\section{La importancia de la salud en la calidad de vida}

Tener salud es un estado físico y mental que le da calidad de vida al ser humano y le permite con ello tener la capacidad para satisfacer sus necesidades básicas. Hoy, con los medios de comunicación y el desarrollo de las nuevas tecnologías médicas, tener salud es un valor y una necesidad del ser humano que debe y puede lograr, además la salud se convierte en estos nuevos tiempos en una preocupación de primer orden para el hombre actual (Montiel, 1993).

\section{El turismo de salud}

El concepto de turismo de salud no es algo novedoso, existe desde los años setenta (San Pedro Martínez, 2004). La finalidad de los primeros balnearios de aguas termales era la recuperación de la salud, sin embargo, el concepto ha cambiado y hoy en día los balnearios representan un lugar para divertirse más que un espacio de salud. La sociedad mexicana está en transformación y cambio, está pasando por un proceso en el cual su nivel cultural está creciendo, ello lleva a un deseo de las personas por cuidar y mantener estable su salud y el cuidado de la naturaleza.

Surge ahora la pregunta ¿qué es el turismo de salud? «Es la acción que se produce al momento en que las personas viajan desde su residencia habitual por razones de salud» (Ross, 2015, p. 1). Existen otros enfoques como el de Gómez, García y Becerril (2013): «La vulnerabilidad en la salud de los viajeros está relacionada con quienes forman parte del fenómeno de movilidad, lo cual impacta en los cambios culturales y sociales, mismos que pueden repercutir en problemas de salud física y emocional» (p. 27). Contrariamente, el turismo de salud implica dirigirse a un lugar que provee bajos riesgos a la salud y, en contraparte ofrece mejorar la calidad de vida a partir de una mejora en la salud del turista o del viajero que acude a un centro de aguas termales. Como señala Sánchez (2006): «Turismo de salud es acudir a un centro donde se ofrece una serie de técnicas para mejorar y equilibrar el estado de salud y bienestar y/o recuperar la salud» (p. 8).

En España el sector de turismo de salud que aprovecha el agua termal tiende a la especialización terapéutica y a la oferta de salud con uso de aguas termales integrando la «salud y bienestar». Destacan los programas preventivos de futuras dolencias para personas predispuestas, preventivos para personas sanas, programas de puesta en forma-belleza y, finalmente, circuitos termales y espacios termolúdicos (Pérez, 2011, citado en Arévalo y Guerrero, 2014, pp. 129-130).

Existen dos enfoques del turismo de salud: la terapéutica y la turística: La primera se vincula más con la medicina que con el turismo, la segunda entiende el turismo de salud desde una perspectiva turística. 


\section{FODA en los balnearios del corredor Ixmiquilpan - Tasquillo}

El estado de Hidalgo se ubica en la zona centro de México. Su superficie es de aproximadamente 20,905 $\mathrm{km}^{2}$, que representan el $1.1 \%$ de la superficie total del país, tiene 2.5 millones de habitantes. Limita al norte con el estado de San Luis Potosí, al noreste con Veracruz, al sureste con Puebla, al sur con Tlaxcala y el estado de México, y al oeste con Querétaro. Por su parte la región Ixmiquilpan - Tasquillo se ubica en la zona central poniente del estado de Hidalgo.

Basados en el sistema de Fortalezas, Oportunidades, Debilidades y Amenazas (FODA) es como se obtiene el diagnóstico. Las fortalezas son las capacidades y habilidades que posee la empresa, las cuales le generan una posición por encima de la competencia. Las oportunidades están representadas por los factores que son favorables y con posibilidad de ser explotables, se encuentran en el entorno de la empresa y le permite obtener ventajas competitivas. Las debilidades son los factores que originan desventaja frente a la competencia, por ejemplo, aquello de lo que se carece (habilidades, actividades, etc.). Las amenazas se originan en el entorno y pueden llevar a la desaparición de la empresa.

Como se observa en el mapa 1, se detecta una fuerte presencia de espacios turísticos a lo largo de toda la parte sur del estado de Hidalgo, de las más importantes por oferta turística y llegada de visitantes se tiene al corredor turístico Ixmiquilpan - Tasquillo; Huichapan - Tecozautla y Huasca.

Los balnearios de aguas termales se ubican principalmente en el corredor Ixmiquilpan - Tasquillo y en Huichapán - Tecozautla, lugares donde se ubican en su mayoría las aguas termales del estado.

Mapa 1: Balnearios de aguas termales en el estado de Hidalgo

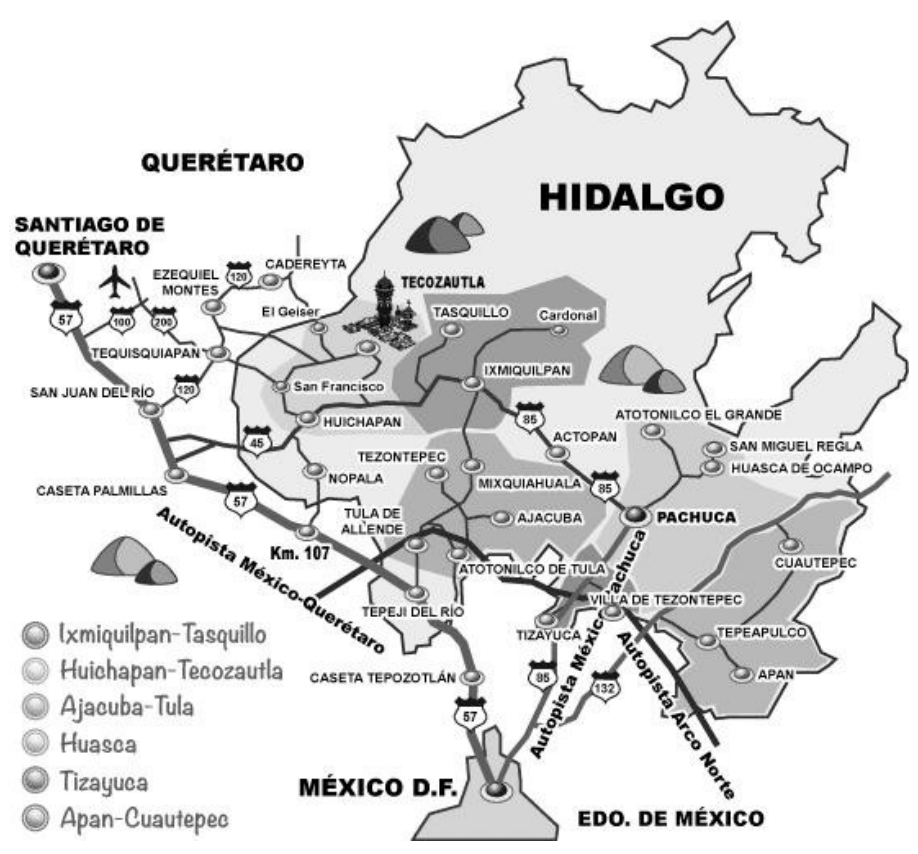

Fuente: Disponible en el enlace http://www.balneariosdeaguastermales.com.mx/ 
La zona de parques de aguas termales en Hidalgo comprende principalmente los siguientes balnearios: Albatros; El Arenal; Chichimequillas; El Geiser; Dios Padre; El Pathecito; Tlacotlapilco; El Tephé; Tepathe.

Con base en un estudio de campo realizado en el corredor Ixmiquilpan - Tasquillo, se logró detectar algunos elementos que nos permiten determinar las fortalezas, oportunidades, debilidades y amenazas de ese corredor de balnearios de aguas termales.

El trabajo de campo tuvo como objetivo detectar: Si el personal que labora dentro de los balnearios ofrece trato cordial; si tienen conocimientos de lo que hacen; si existe una atención personalizada; si los empleados hacen su actividad con empeño; si existen precios competitivos en los productos ahí vendidos; si hay limpieza e higiene en los baños, albercas, restaurantes y hoteles; si el equipamiento es moderno; si existe asistencia médica tanto en las piscinas como en el hotel; si son adecuadas las actividades complementarias que ofrece el parque; si existen actividades complementarias que permitan aprovechar las aguas termales con métodos terapéuticos y de belleza; si existe en los hoteles reserva garantizada a través de internet; si el entorno natural en el cual se ubica el parque es adecuado; si existe infraestructura moderna, si la ubicación geográfica le hace ser un lugar factible para la llegada del visitante y turista; si los servicios complementarios son eficientes, si los programas de salud son adecuados a las necesidades de la gente; si los precios de entrada no laceran el bolsillo de las familias y, si existe seguridad dentro del parque y en los estacionamientos de los balnearios. Con base en la visita in situ realizada y con los resultados de las cuarenta entrevistas aplicadas, se logró detectar lo siguiente:

\section{Fortalezas}

a. Existencia de una gran variedad de parques acuáticos en la zona en un radio no mayor a 20 kilómetros. El visitante tiene la oportunidad de elegir por una u otra opción de acuerdo a sus gustos y preferencias.

b. Oportunidad al visitante para elegir dentro de la abundante oferta el mejor lugar con base en precio y calidad de los servicios. El 75\% de los entrevistados aceptó haber tenido buenas opciones para elegir y decidir la mejor decisión.

c. El 95\% de los entrevistados afirmó haber revisado por la web todos los parques existentes en la región, lo cual les permitió explorar desde casa las diferentes opciones que el corredor Ixmiquilpan - Tasquillo ofrece en balnearios de aguas termales.

d. Los parques no solo ofrecen albercas con aguas termales, cuentan en su gran mayoría con toboganes, riachuelos artificiales; albercas con olas; habitaciones en hoteles de 2 y 3 estrellas; zona de acampar, restaurantes, palapas, etc.

e. Su ubicación geográfica estratégica la ubica a 75 kilómetros de la ciudad de Pachuca, capital del estado de Hidalgo, a 66 kilómetros de la ciudad de México y a 150 kilómetros de la ciudad de Querétaro.

f. Agua limpia en todas las piscinas, de hecho en algunos balnearios el agua se cambia todos los días.

g. La llegada a todos los balnearios es por carretera pavimentada. 
h. El agua es termal, lo cual además de sus propiedades curativas hace que el visitante disfrute de agua a temperaturas entre 25 y 40 grados.

i. El clima de la zona es cálido, oscila durante el año entre los 11 y 35 grados centígrados promedio anuales.

j. El 80\% de los entrevistados afirmó que el precio de entrada al balneario con base en la oferta de servicios es adecuada, además se detectó que los balnearios ofrecen precios diferenciados, los cuales dependen del tamaño del balneario y de los servicios de infraestructura que ofrecen.

k. Hay tarifas preferenciales para niños y personas de la tercera edad.

1. Existe la posibilidad de acampar, lo cual hace más económica la estancia si se desea pasar ahí todo el fin de semana.

\section{Oportunidades}

a. Existen amplios espacios para incrementar dentro de los balnearios los atractivos y ofertas de servicios.

b. Posibilidad de difundir dentro de los balnearios las bondades y beneficios de las aguas termales, lo cual es casi inexistente actualmente.

c. Ofrecer hospedaje con categoría 4 y 5 estrellas.

d. Ofrecer a los visitantes y turistas la posibilidad de conocer espacios en diferentes lugares del municipio, esto es, que la visita del turista no solo sea el parque, sino que también se dirijan a otros espacios turísticos dentro del municipio o la región.

e. Posibilidad de hacer del balneario una oferta de productos integrados, más allá del uso de albercas y toboganes.

f. Posibilidad de que los hoteles dentro de los balnearios ofrezcan actividades recreativas a sus huéspedes durante el transcurso de la noche.

g. Difundir la oferta de balnearios a través de un mayor uso de los medios de comunicación. El 95\% de los entrevistados afirmó haber visto los balnearios por páginas web de los propios balnearios, sin embargo, no lograron ver o escuchar la oferta turística por otras opciones como radio, periódicos o televisión local o nacional.

\section{Debilidades}

a. El 100\% de los entrevistados se quejó por no haber logrado reservar la habitación del hotel a través de internet.

b. El 100\% de los entrevistados afirmó que el precio de entrada es excesivo si se desea pernoctar en alguno de los hoteles dentro del balneario, esto es, se debe pagar el ingreso al balneario, la noche de hospedaje en el hotel y el segundo día dentro del balneario. Lo anterior hace elevado el costo total para que una familia se divierta durante el fin de semana. 
c. Falta de personal, lo cual dificulta que en muchas ocasiones se logre resolver dudas de la ubicación de baños, restaurantes, hoteles y espacios de diversión. En este sentido el 72.5\% afirmó lo anterior, solo el $27.5 \%$ estuvo de acuerdo con el servicio de orientación que da el balneario.

d. El 90\% de los visitantes y turistas afirmó que el personal de los hoteles carece de capacitación y buen trato, se percataron de la existencia de incapacidad hacia el turista y visitante, no había respuestas claras y precisas, por ejemplo, al momento de solicitar una habitación se les informó que no existían disponibles, se les preguntó si sabían de otro hotel dentro del balneario que tuviera disponibilidad, la respuesta fue no, pero tampoco les ofrecieron apoyo alterno.

e. Para el $87.5 \%$ de los visitantes y turistas, consideran que algunos de los balnearios necesitan mantenimiento, particularmente en los baños y regaderas, estas se encuentran en mal estado, sucias y sin infraestructura para por ejemplo, colgar una toalla junto a la ducha.

f. Para el $70 \%$ de los turistas y visitantes existe insatisfacción en los servicios, destacan: mala calidad en la atención al cliente, particularmente en los servicios de alimentación y hospedaje.

g. El $67.5 \%$ detecta la escasez de actividades lúdicas alternativas, ello les genera tedio y aburrimiento, para ellos y ellas las estancias cortas de un día son las que prefieren.

h. Se detecta en el trabajo de campo que recibir visitantes y turista en los destinos observados es desordenado, no hay capacitación en los que atienden a los visitantes, no comprenden lo que es el servicio al cliente con características de calidad.

i. Para el $95 \%$ de los turistas y visitantes la seguridad al interior de los balnearios sí es adecuada, sin embargo, de ese $95 \%$, el $35 \%$ detectó la falta de salvavidas, principalmente entre semana.

\section{Amenazas}

a. La posibilidad que en un futuro lejano las aguas termales de la zona dejen de existir.

b. La competencia agresiva que pudieran generar los balnearios de aguas termales ubicados en el estado de Querétaro o en el corredor Huichapán - Tecozautla.

\section{Retos y acciones para fortalecer el turismo de salud en la región de estudio}

Con base en el FODA detectamos la existencia de una serie de retos y acciones. Proponemos las siguientes:

a. Se requiere aumentar el flujo turístico, especialmente en temporada baja. Ello, a través de campañas agresivas de comunicación local y regional acompañadas de bajos precios para el ingreso, y es que se observó entre semana aproximadamente el $10 \%$ de afluencia de visitantes a lo observado durante los días de fin de semana.

b. Es necesario atender otros segmentos de mercado, como ya señalamos, el turismo de salud.

c. Capacitación del personal que se encuentra vinculado al turismo. 
d. Estimular la creación de infraestructuras básicas y servicios para el desarrollo de otras actividades.

e. Modernizar y mejorar la calidad de los establecimientos.

f. Fomentar la coordinación entre balnearios y de estos con la administración pública municipal.

g. Lo justo sería pagar el hospedaje el cual incluya los dos días de estancia en el balneario a un precio preferencial, por ejemplo, pagar una noche de hospedaje más el primer día de estancia dentro del balneario, el segundo día puede ser gratis.

h. Es necesario que la mejora de competitividad del sector de aguas termales superen el concepto tradicional de balneario como centro de diversión y ofrezcan el servicio adicional como centro de salud, lo cual vaya acompañado de la oferta de productos y servicios complementarios a la actividad lúdica de aguas termales.

i. Existen experiencias internacionales las cuales muestran que los balnearios ofrecen servicios diferenciados y heterogéneos, por ejemplo, hay espacios especializados en atender a personas de la tercera edad, tratamientos antiestrés y de belleza; actividades antes señaladas que no se logran percibir en ninguno de los balnearios visitados.

j. Dada la vida tan ajetreada que lleva la sociedad actual, los balnearios se deben transformar y por ende ofrecer más y mejores servicios, por ejemplo, relajación antiestrés, programas de adelgazamientos, estético y de belleza, rejuvenecimiento o revitalización, programas de relajación de fin de semana, etc.

k. Para ofrecer los servicios es necesario elaborar un programa de marketing con el objeto de publicitar los servicios y venderlos principalmente en las páginas web de turismo.

1. El espacio termal debe ser la principal fuente de atracción, pero además se debe ofrecer como atractivo secundario la visita a los municipios en los cuales se encuentran enclavados los balnearios, por ello, los municipios deben representar para los turistas un espacio de esparcimiento complementario al balneario.

m. Con la nueva competencia existente entre los oferentes de servicios turísticos, es necesario considerar el balneario como un espacio completo en el cual se ofrezca la recreación, la parte de la salud termal y el hospedaje. Como argumenta González (2003): «Lo que se pretende con este tipo de actividades es hacer del producto termal un producto integrado por varias categorías de servicios, entre ellos una amplia oferta de ocio, que consigan la diferenciación de la oferta del establecimiento» (p. 244).

n. Los balnearios que ofrecen hospedaje, deberán brindar por la noche servicios complementarios, por ejemplo, espectáculos nocturnos que hagan que se aumente el atractivo del destino y por ende la llegada de visitantes.

o. Los avances tecnológicos en los medios de comunicación, la revolución en los medios de transporte y los estudios y difusión de los beneficios de las aguas termales apoyan el desarrollo de los balnearios de aguas termales. Actualmente las personas visitan los balnearios principalmente para relajarse y divertirse, sin embargo, muchos de ellos consideran como un valor extra la mejora de la salud (Costa, 1999; San José, 2000). 


\section{Conclusiones}

En la actualidad los balnearios de aguas termales se encuentran en un continuo proceso de expansión. En el mundo los balnearios siguen una tendencia mundial por la búsqueda de una mejor calidad de vida, ello a través de la oferta de tratamientos termales los cuales ofrecen una salud integral.

La zona de aguas termales objeto de estudio, muestran que una de las características de la demanda turística, es que los visitantes van con la motivación de disfrutar del parque temático. Sin embargo, ya lo señalamos a lo largo del texto, se debe reorientar el servicio hacia el turismo de salud y de belleza.

Los balnearios tradicionales realizan actividades y servicios como alojamiento, gastronomía y actividades de ocio, sin embargo, no consideran otras ofertas que hagan más atractiva la visita a dichos centros, por ejemplo, la existencia de espacios de cuidados de belleza y actividades de mantenimiento físico.

La importancia de la calidad en los servicios es fundamental y, es que mejora la satisfacción del cliente y por otra parte aumenta la comunicación de difusión y de promoción boca en boca, en turismo es un criterio importante de elección la recomendación de familiares y amigos.

Consideramos que los balnearios del corredor Ixmiquilpan - Tasquillo no cuentan con los servicios secundarios que les permita ser explotados con eficacia y eficiencia. Actualmente los balnearios deben pensar en las necesidades del nuevo consumidor. Se deben acercar los balnearios hacia otros productos del turismo de salud como los spas, resorts, etc., sin embargo, el principal insumo es el agua minero medicinal. Se debe considerar de forma permanente lo que el producto ofrece a sus clientes. Pensar en el cambio y en la innovación permanente $y$, es que las nuevas necesidades y deseos de la demanda de hoy, es diferente a lo que se demandaba ayer. 


\section{Referencias}

Arévalo, G. J. \& Guerrero, H. R. (2014). Turismo de salud por medio del aprovechamiento de aguas termales. Caso de la Ruta de la Salud Michoacán. Economía y sociedad 28(31), 121-153.

Balderas, R. (2014). Global Conference on Business and Finance. Proceedings Institute for Business \& Finance Research, 9(2), 1630-1634.

Brown, J. \& Canter, D. (1985). The uses of explanation in the research interview. En M. Brenner et al. The research interview. Uses and approaches (pp. 76-98). London: Academic Press.

Costa, A. (1999). Turismo de salud en 50 años del turismo español. En M. Bayón (coord.), 50 años de turismo español: un análisis histórico y estructural (pp. 737-747). Madrid: Universitaria Ramón Areces.

Chadwick, B., Barch, H., \& Albretch, S. (1984). Social science research methods. Prentice Hall. Englewood Cliffs.

Fernández, L. (1991). Geografia General del Turismo de Masas. Madrid: Alianza Editorial.

Gómez, D., García, C., \& Becerril, P. (2013). Cuidando la salud de los viajeros. Un encuentro con la Emporiatría. El Periplo Sustentable. Recuperado de <http://rperiplo.uaemex.mx/index.php/elperiplo/article/view/676/494>

González, M. A. E. (2003). Análisis de la calidad de servicio percibida en los establecimientos termales: conceptualización, medición y relación con otras variables de marketing. (Tesis doctoral). Facultad de Ciencias Empresariales de Ourense, Universidad de Vigo.

Kane, T. (1985). Doing your own research. Basic descriptive research in the social sciences and humanities. London: Marion boyars.

Mitchel, R. (1973). Survey material collected in the developing countries: sampling, measurement, and interviewing. Obstacles to intra an international comparisons. En E. Warwick, Comparative research methods. Englewood Cliffs: Prentice Hall.

Montiel, L. (1993). Beneficios y riesgos de un nuevo valor: el valor salud en la sociedadpostindustrial. En L. Montiel (coord.), La salud en el estado de bienestar: análisis histórico. Cuadernos complutenses de historia de la medicina y de la ciencia (pp. 3753). Madrid: Complutense.

Ortíz, P. et al. (2014). Turismo como detonante de desarrollo local regional en la ruta de la salud, Michoacán. Revista Nicolaita de Estudios Económicos, 9(2), 107-137.

Ross, K. (2015). Health Tourism: an overview. Recuperado de http://www.hospitalitynet.org/news/4010521.html.

San José, C. (2000). Guía médica de los balnearios de España. Sevilla. Sevilla: Universidad de Sevilla.

Sánchez, A. (2006). Turismo de salud: situación actual y perspectivas de futuro. Ourense: Universidad de Vigo.

San Pedro Martínez, M. A. (2004). El turismo de salud. En Asociación Española de expertos científicos en turismo (ed). La actividad turística española (pp. 559-574).

Somer, R. (1986). A practical guide to behavioral research tools and techniques. New York: University Press. 


\begin{abstract}
ANEXO I
Metodología de la entrevista

A la salida de los balnearios se realizaron 40 entrevistas a mismo número de visitantes y turistas dentro de los balnearios del corredor Ixmiquilpan - Tazquillo (se omitirá el nombre de los balnearios para evitar ponerlos en evidencia). Las entrevistas se realizaron durante seis diferentes visitas a los centro recreativos, se acudió al destino tanto en días entre semana como en días de fin de semana, ello con el objeto de tener una mejor panorámica del centro recreativo.
\end{abstract}

Consideramos que la entrevista es un instrumento más apropiado para tratar el tema en estudio, en virtud de la interacción personal y la mayor profundidad cualitativa que se obtiene al entrevistar personas.

Si bien es cierto, sabemos que hay limitaciones de las entrevistas para estudiar las actitudes de los individuos, no obstante, las entrevistas son más apropiadas cuando se estudian datos de comportamiento de primera mano. Así, se consideró que una entrevista servía mejor para los propósitos y las necesidades de investigación de este artículo (Chadwikc, Barch y Albretch, 1984).

La segunda decisión fue determinar si la entrevista sería estructurada (programada) o no estructurada (guiada) y el orden de las preguntas, el formato (cerrado o abierto) y el orden de las preguntas.

Existen argumentos a favor y en contra de ambos tipos de entrevistas. La primera es más apropiada para probar hipótesis específicas y cuantificar con rigor y sentido los resultados derivados de los diferentes interlocutores (Chadwick et al., 1984).

Las entrevistas programadas facilitan el manejo y el análisis de los datos y reducen la probable parcialidad encontrada en entrevistas menos estructuradas, así como el riesgo de obtener excesivo material irrelevante (Mitchel, 1973; Somer, 1986). Por otra parte, las entrevistas no estructuradas proporcionan información más cualitativa y detallada sobre asuntos complejos, y son útiles estos estudios exploratorios para desarrollar investigación más estructurada. Existe consenso en que una entrevista completamente no estructurada y sin dirección es inapropiada para la investigación, dado que incrementa enormemente el trabajo de análisis. Así, un enfoque semiestructurado es más adecuado (Brown y Canter, 1985; Kane, 1985).

Las entrevistas pueden ser guiadas y todavía centrarse en grandes tópicos de interés. Como se argumenta: «La investigación seria más eficaz si los procedimientos permitieran a los entrevistados expresar sus propios puntos de vista al tiempo que proporcionan información lo suficientemente estructurada para el análisis y el reporte sistemático» (Brown y Canter, 1985).

Por lo antes señalado se decidió que en la investigación se usara un formato semiestructurado que incluyó preguntas específicas acerca de tópicos de interés previamente determinados, la entrevista fue conducida personalmente, la cual incluyó preguntas centradas en tópicos relevantes a los asuntos investigados y dirigidos a personas que hayan pasado horas o días dentro de los balnearios. 
Se determinó que el orden de las preguntas ira de la menor a la mayor importancia. Existe consenso de que en general las preguntas interesantes y relativamente fáciles de contestar deberían aparecer al principio. Una vez que se ha establecido cierta confianza con el entrevistado (Somer, 1986; Chadwick et al., 1984). En total se realizaron 17 preguntas, las cuales anotamos a continuación:

1. ¿Considera Usted que dentro del corredor Ixmiquilpan - Tasquillo existe una oferta de opciones de balnearios suficiente o insuficiente para elegir la mejor opción?

2. ¿Pudo ver por internet las diferentes opciones de balnearios ofrecidos en el corredor turístico Ixmiquilpan - Tasquillo?

3. ¿Considera adecuado o inadecuado el precio con base en los servicios recibidos dentro del balneario?

4. ¿Encontró tarifas preferenciales para niños y personas de la tercera edad?

5. ¿Se le ofreció servicios turísticos atractivos dentro del municipio?

6. ¿Dentro del balneario se les ofreció o detectó servicios de salud termal?

7. ¿Cómo considera la calidad de los servicios de alimentación y hospedaje dentro del balneario? Con base en las siguientes opciones (buena, mala, muy buena, muy mala, regular)

8. ¿Realizó actividades lúdicas que le permitieron mantenerse distraído durante toda su estancia en el balneario?

9. ¿Considera a los empleados del balneario como personal capacitado para realizar sus funciones?

10. ¿La seguridad en el balneario fue buena, mala, regular?

11. ¿Considera que el balneario necesita mantenimiento en sus instalaciones?

12. ¿Se hospedó dentro de alguno de los hoteles del balneario? Si su respuesta es sí, conteste las siguientes preguntas:

13. ¿Dentro del hotel se le ofreció algún espectáculo nocturno?

14. ¿Dentro del hotel se le ofreció o encontró alguna actividad lúdica?

15. ¿Tuvo la posibilidad de reservar el hotel a través del internet?

16. ¿Considera que el pago por la noche de hospedaje, más el pago por dos días de estancia dentro del balneario es excesivo o le parece un precio justo?

17. ¿Regresaría a hospedarse y a la vez pagar por dos días de estancia en el balneario. Si su respuesta es sí/ no, ¿Por qué? 